Technical note

\title{
Corrective limb osteotomy using patient specific 3D-printed guides: A technical note
}

\author{
H. Hoekstra, Prof. MD FEBS ${ }^{a, b, *, 1}$, W. Rosseels ${ }^{c, 1}$, A. Sermon, Prof. MD ${ }^{a, b}$, S. Nijs, Prof. MD ${ }^{a, b}$ \\ a University Hospitals Leuven, Department of Trauma Surgery, B-3000 Leuven, Belgium \\ ${ }^{\mathrm{b}} \mathrm{KU}$ Leuven-University of Leuven, Department of Development and Regeneration, B-3000 Leuven, Belgium \\ ${ }^{\mathrm{c}}$ KU Leuven-University of Leuven, Faculty of Medicine, B-3000 Leuven, Belgium
}

\section{A R T I C L E IN F O}

Keywords:

3D-guiding

Corrective osteotomy

Malunion

\begin{abstract}
A B S T R A C T
We describe the step-by-step process of a corrective osteotomy using 3D printed patient specific guides. Before surgery, bilateral computed tomography (CT) scans are made to plan correction in the affected limb. The digital pre-planning defines the location of the K-wires, drill holes, and the osteotomy site(s). Subsequently, a 3D printed patient specific guide is applied, which indicates the exact position of these drill holes and the osteotomies. This increases the accuracy of the surgery by means of patient specific fit of the guide. During surgery an incision is made and the guide is applied on the bone, which allows the surgeon to perform a very precise osteotomy. Next, the bone is reduced either directly using the plate and marked drill holes, or indirectly using a second reduction guide. In the latter case, the previously drilled $\mathrm{K}$-wires are used to adequately position the reduction guide. Fixation of the bone fragments using plating osteosynthesis finalizes the process. Although this technique has its specific limitations, it might serve as a powerful tool in the treatment of malunion of both articular and nonarticular fractures of the limb.
\end{abstract}

(c) 2016 Elsevier Ltd. All rights reserved.

\section{Introduction}

During the 1980s, a whole new technique to accurately reconstruct physical objects was developed: 3 dimensional (3D) printing. Ever since, this technique is used for a wide range of applications. In the beginning the majority of its medical application has been in the field of reconstructive maxillofacial surgery and dental surgery [1-3]. Whereas the use of this technique in trauma surgery is still in its infancy [4]. However, some promising results might be achieved with this technique, for example, during corrective osteotomies, a procedure in which bone anomalies resulting from a prior trauma are corrected by cutting bone. This kind of surgery can be very complex and challenging. Underestimating the blurred deformity lines during surgery may result in a less accurate performance of the preoperative surgical plan and a less predictable outcome. In these cases, the technique of 3D planning and printing helps the surgeon to tackle such difficulties, since it allows the surgeon to visualize the anatomy in full 3D and to digitally plan the osteotomy

\footnotetext{
* Corresponding author at: University Hospitals Leuven, Department of Trauma Surgery, Herestraat 49, B-3000 Leuven, Belgium.

E-mail address: harm.hoekstra@uzleuven.be (H. Hoekstra).

1 Both authors contributed equally.
}

preoperatively based on patient's CT images, taking multiple surgical approaches into account. Therefore, a patient specific surgical guide is designed to guide the cutting and reduction according to the surgical plan, hereby improving the predictability of osteotomy procedures [5-8].

Hence, 3D printing may serve as a tool for a better understanding of complex fracture patterns resulting in increased accuracy of preoperative planning, increased precision of surgical navigation, decreased postoperative complication ratio, a more cost-effective use of operating rooms, as well as improvement of patient satisfaction [8,9]. This technical note presents a step-bystep description of the process of 3D guided osteotomies for malunion of both articular and nonarticular limb fractures, based on our experiences in more than a dozen cases.

\section{Process overview}

1. Adequate imaging of the malunion and osteotomy site is the important first step in generating 3D printed guides (Fig. 1).

2. Based on CT imaging, virtual 3D models are created for both deformed and contralateral side (Materialise Interactive Medical Image Control System Software, Materialise, Leuven, Belgium). The desired mechanical variables (rotation, length, and both coronal and sagittal alignment) are calculated and the mirrored view of the 


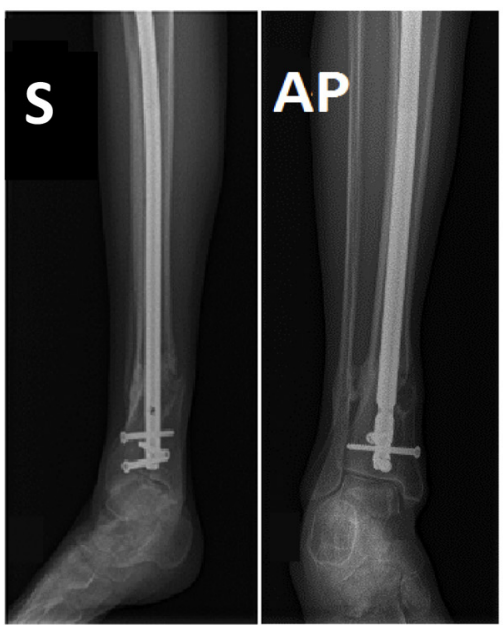

A
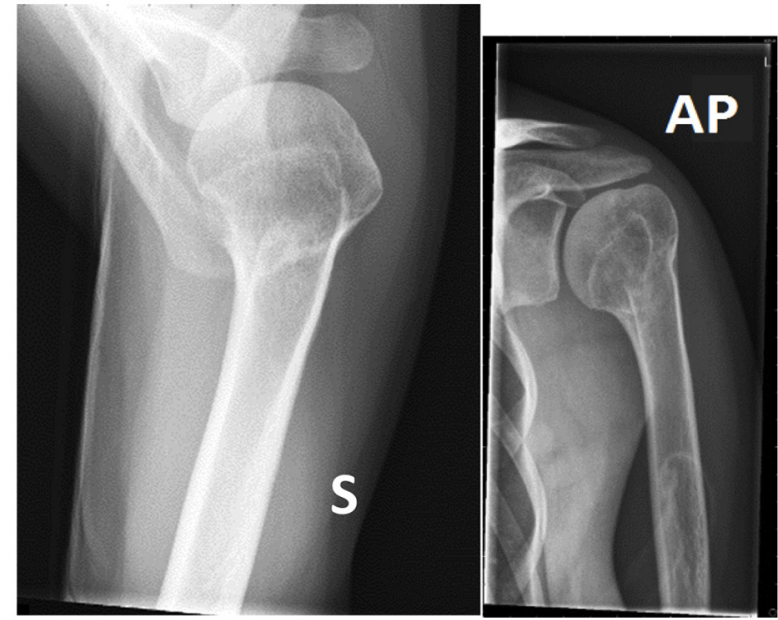

B

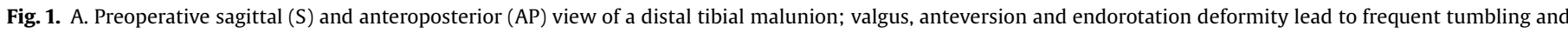

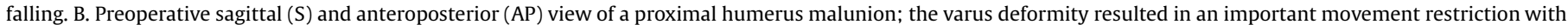
reduced internal rotation and abduction of the left humerus and limited left shoulder flexion.

contralateral limb can be placed over the surgical side as an anatomical reference (Fig. 2).

3. The surgeon decides the position and orientation of the osteotomy. Whether to perform an opening or a closing wedge osteotomy is mainly based on the direction and complexity of the fracture pattern and its anatomy. A virtual example of the osteotomy is performed, during which the above mentioned template is superimposed as a transparent model. Next, a virtual model of the plate is made and placed on the corrected bone model. Size and position of the plate and screw locations are verified by the surgeon (Fig. 3 ).
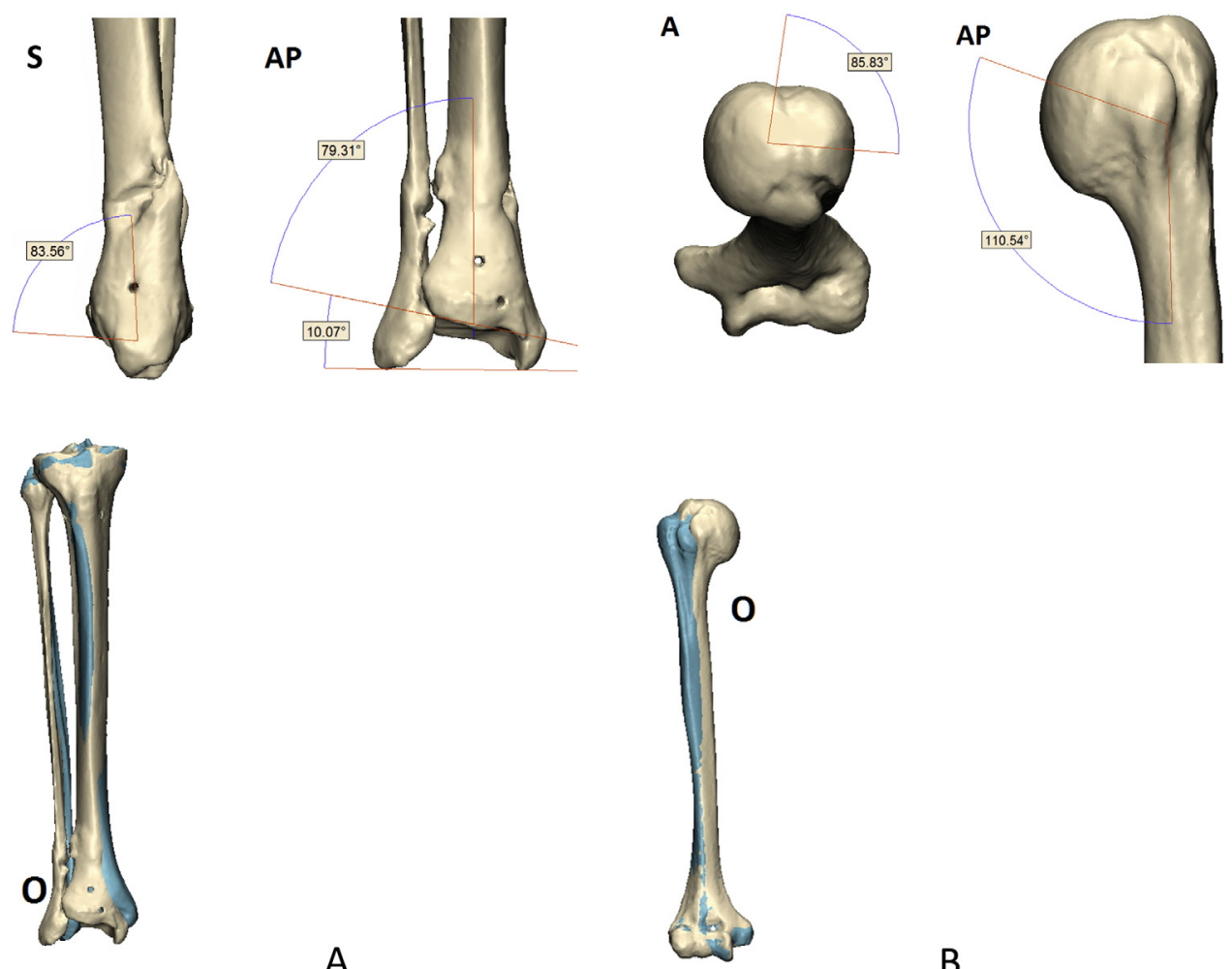

A

B

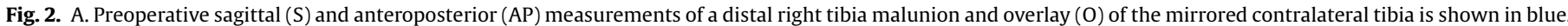

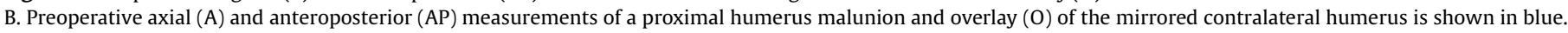
(For interpretation of the references to colour in this figure legend, the reader is referred to the web version of this article.)

Please cite this article in press as: H. Hoekstra, et al., Corrective limb osteotomy using patient specific 3D-printed guides: A technical note, Injury (2016), http://dx.doi.org/10.1016/j.injury.2016.07.021 

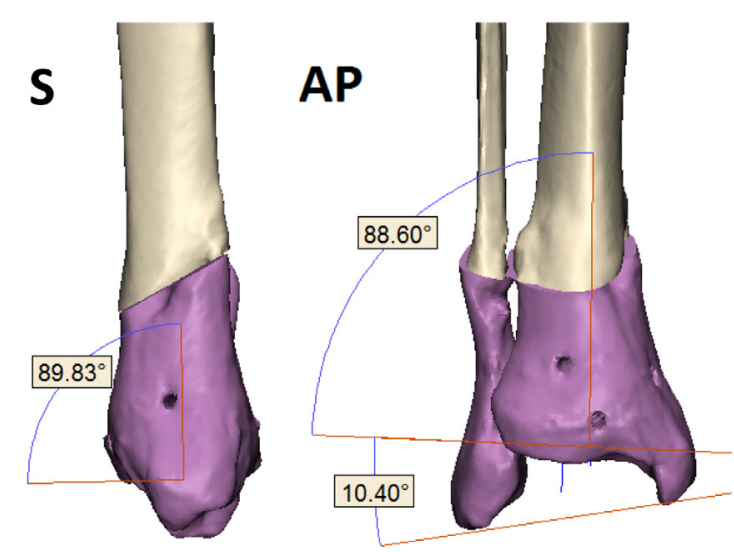

A

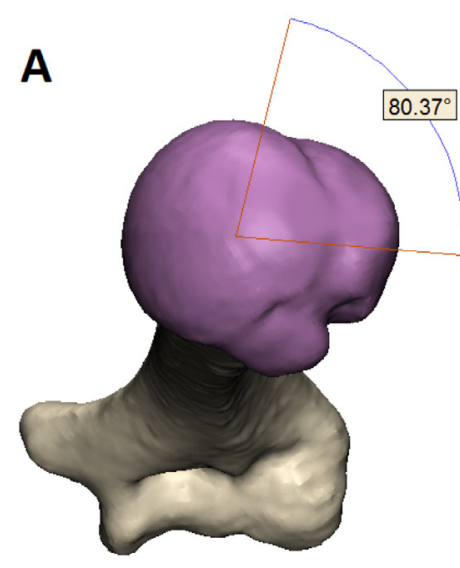

C
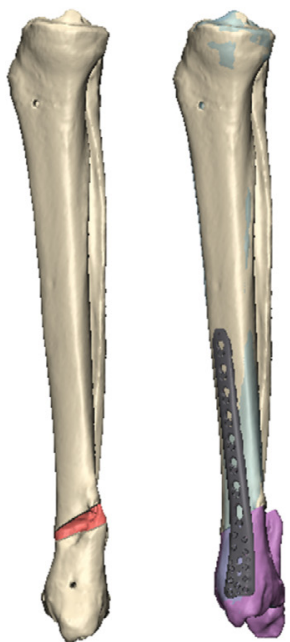

B

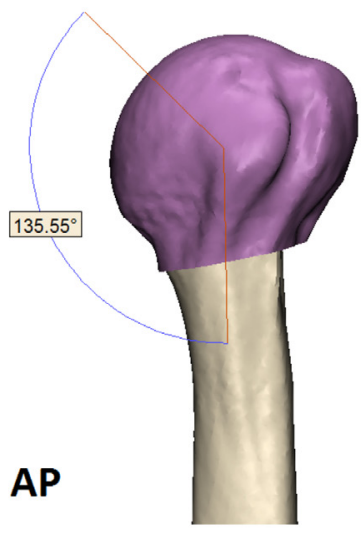

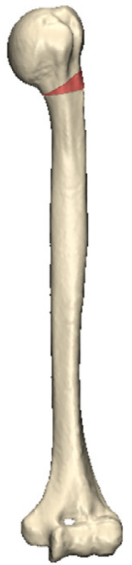

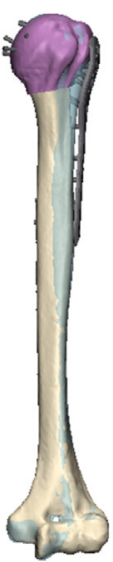

D

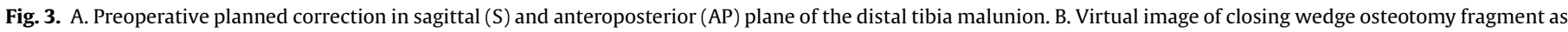

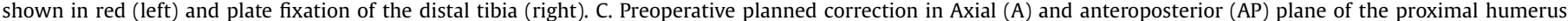

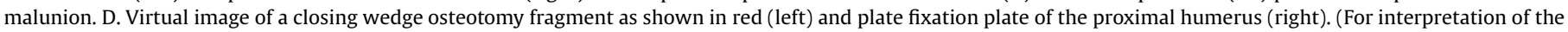
references to colour in this figure legend, the reader is referred to the web version of this article.)

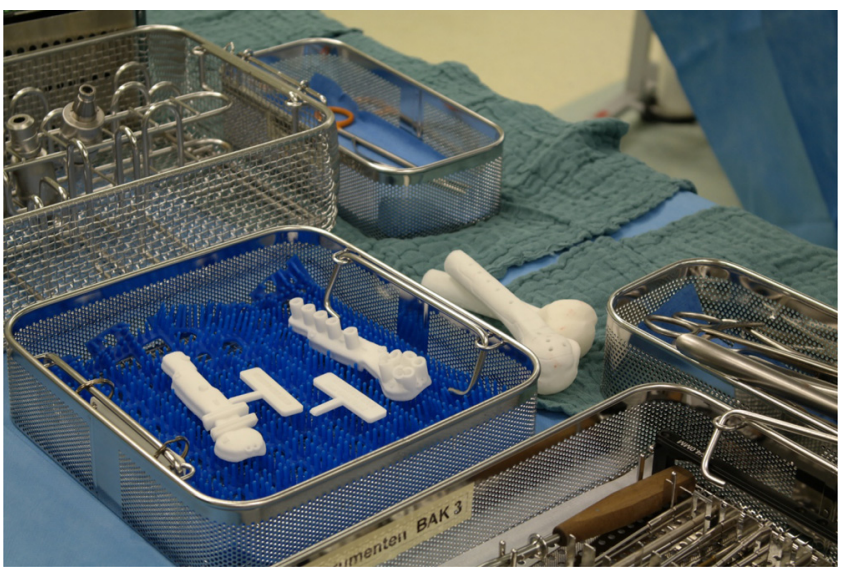

Fig. 4. 3D-printed guides and bone fragments for proximal left humerus.
4. In the final step of preoperative planning, a physical model of the patient specific guide is being printed and labeled together with physical models of the affected bone before and after osteotomy (Fig. 4).

5. Guide positioning and fixation. After verification of adequate fitting of the guide on the bone model, an incision is made and fracture site is exposed. Soft tissue is removed to obtain optimal exposure of the fracture site for a precise positioning of the guide, preferably no gaps between the guide and the underlying bone. Finally, the guide is secured with K-wires (Fig. 5) and, if necessary, screw holes are drilled serving as landmarks for the reduction.

6. Osteotomy. Specially designed cutting slots on the guide are used to optimize the position of the saw blade during osteotomy (Fig. 6). Irrigation of the osteotomy is essential to avoid excessive heating and necrosis of the surrounding soft tissue.

7. Reduction. The bone is reduced either directly using the plate that is fixated by screws in the previously drilled holes, or indirectly using a second guide for reduction. The previously drilled K-wires are used to adequately position this reduction guide (Fig. 7).

Please cite this article in press as: H. Hoekstra, et al., Corrective limb osteotomy using patient specific 3D-printed guides: A technical note, Injury (2016), http://dx.doi.org/10.1016/j.injury.2016.07.021 


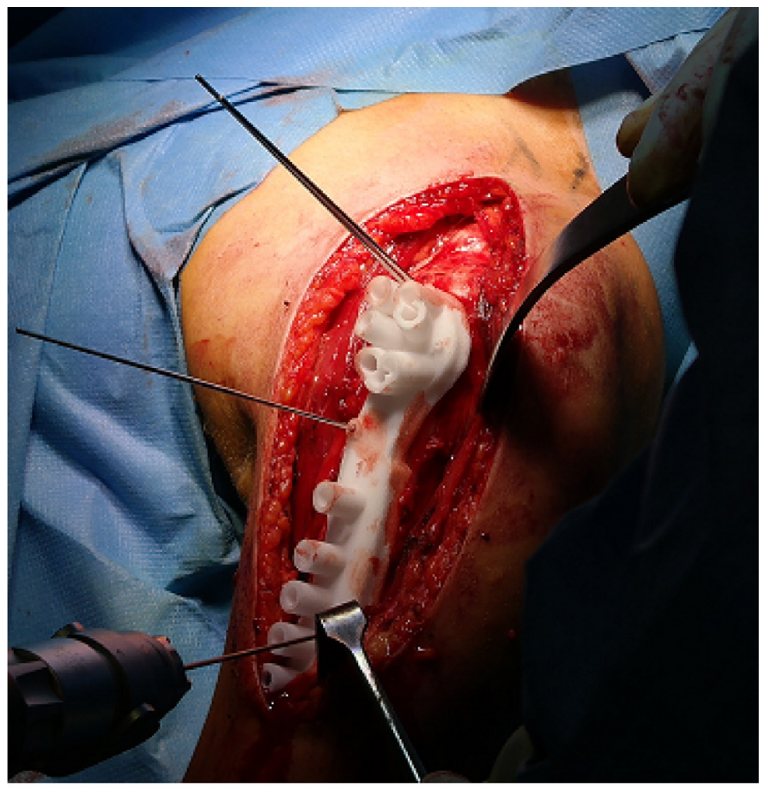

A

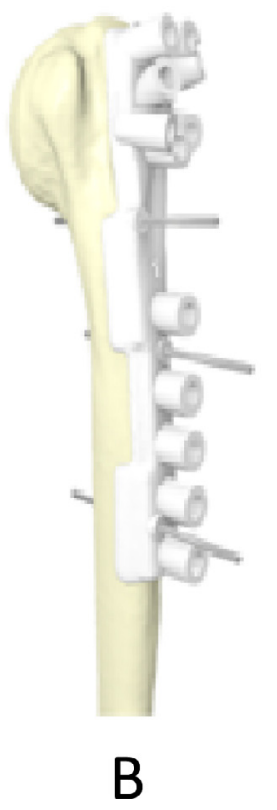

Fig. 5. A. Fixation of the guide with K-wires on the proximal humerus. B. Virtual example of 3D printed osteotomy guide for treatment of proximal humerus malunion.

8. Osteosynthesis. During the final step of the surgical process, a plate osteosynthesis is performed in order to compress the bone parts (Fig. 8).

9. A postoperative CT-scan is made for internal control where the preoperative goals can be compared with the actual results (Fig. 9) and functional outcome (Fig. 10).

\section{Discussion}

In our experience and with regard to the results, osteotomies performed using 3D printed patient specific guides, seem like a promising tool for the treatment of an upper or lower limb malunion. As shown here, it increases the surgical precision and accuracy of preoperative planning because multiple approaches

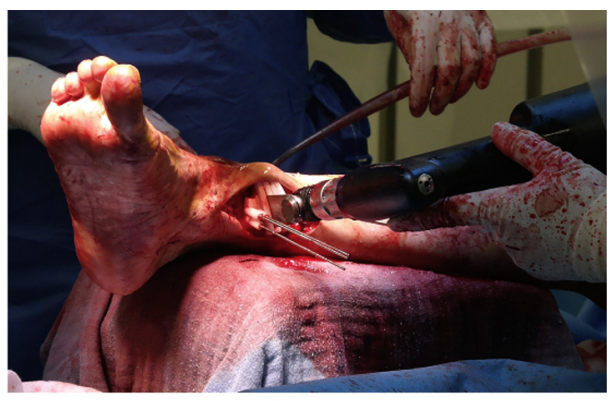

A

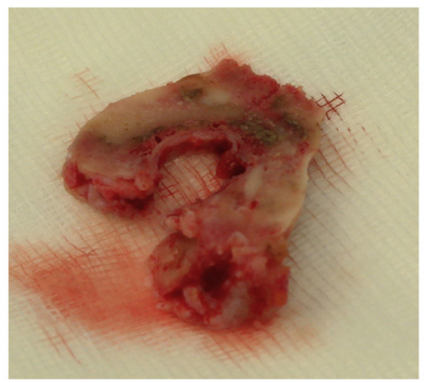

B

Fig. 6. A. Saw blade in guide in situ for the distal tibial osteotomy. B. Osteotomy fragment of distal tibia.

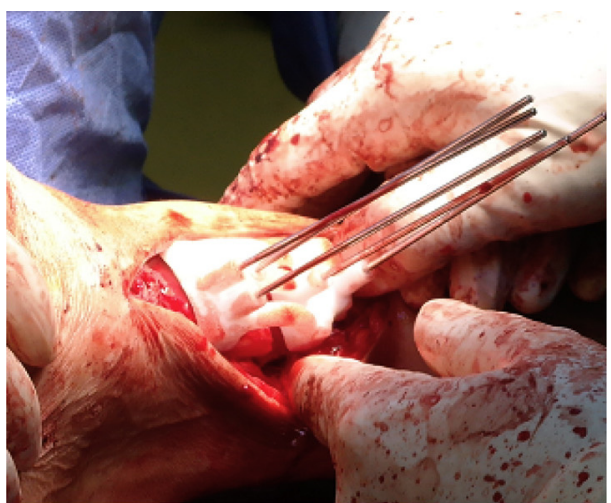

A

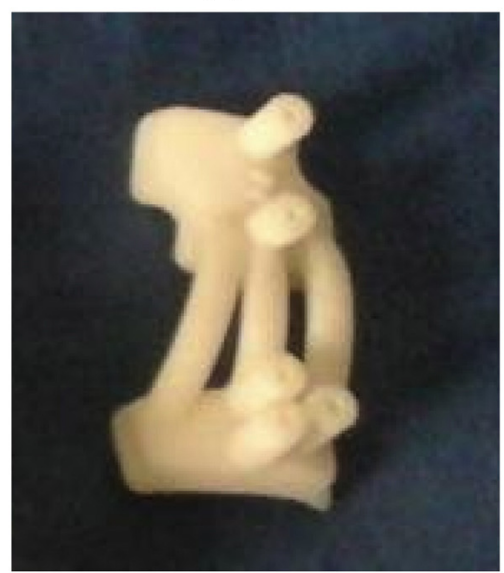

B

Fig. 7. A. Positioning of the reduction guide on the distal tibia with the use of Kwires. B.The 3D printed reduction guide.

Please cite this article in press as: H. Hoekstra, et al., Corrective limb osteotomy using patient specific 3D-printed guides: A technical note, Injury (2016), http://dx.doi.org/10.1016/j.injury.2016.07.021 


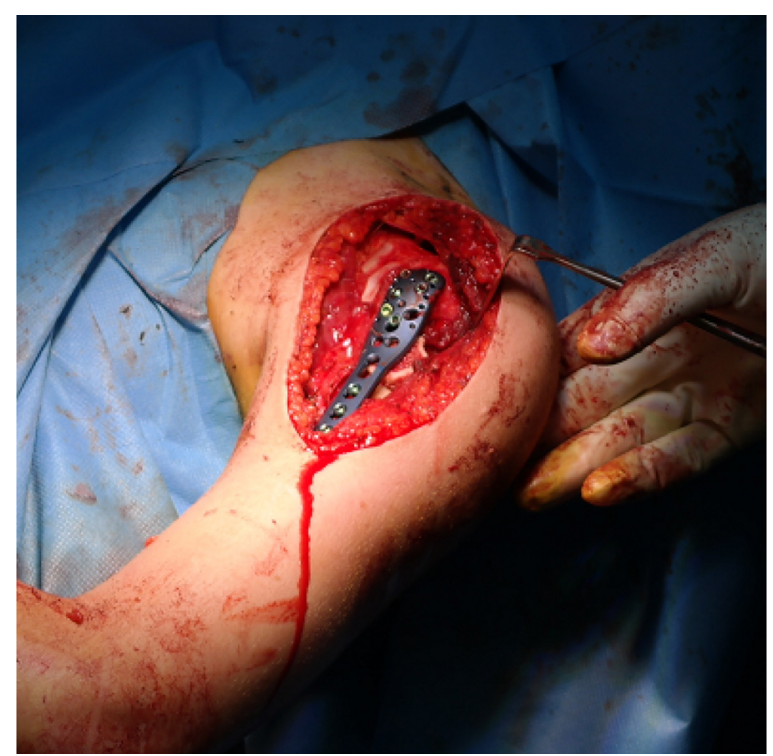

Fig. 8. Plate osteosynthesis of the proximal humerus. and strategies can be taken into account. Until now, with the limited use of software that calculates the desired mechanical variables, it was the sound judgement of the surgeon to weigh different approaches to achieve a satisfactory result.

However, this technique also has its limitations. The exact positioning of the guide is accomplished by adequate exposure of the guide to the circumference of the bone, as expressed by a ratio. Therefore, the use of larger guides while maintaining an optimum ratio, may lead to more than desirable soft tissue dissection. The comprised soft-tissues in turn, affect the occurrence of postoperative wound complications (e.g. infections). Also the periosteum and hereby cortical blood flow might be affected. Moreover, the exposure of the guide to the circumference of the bone, is limited to certain anatomic boundaries.

As with non-guided osteotomies, the reduction remains the main limiting step. Although the reduction is facilitated by predrilled holes or the use of a second reduction guide, applying adequate compression while maintaining the reduction can be hard. Particularly in case of an oblique osteotomy, compression may lead to shifting of bone fragments and loss of reduction.

Furthermore, there may be a discrepancy between the preoperative calculations and the in situ handling. While bone is dynamic tissue, the 3D printed guides are rather static which may result is lack of compression (non-articular osteotomy) or loss of reduction
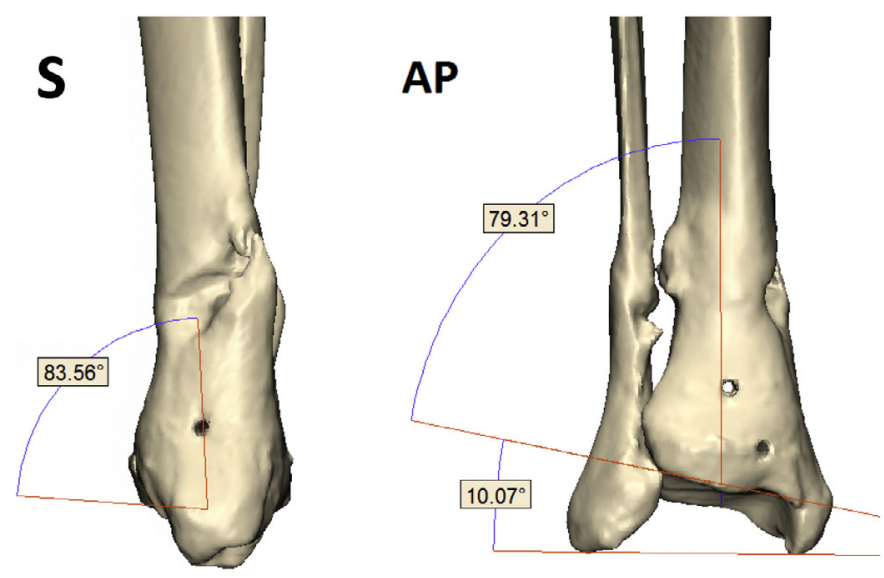

A
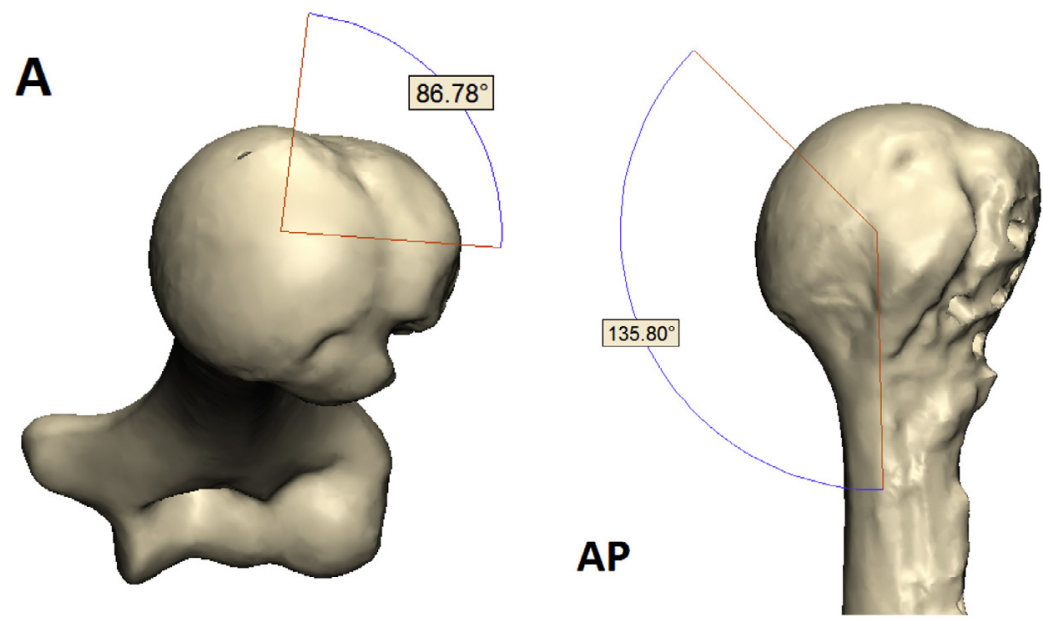

B

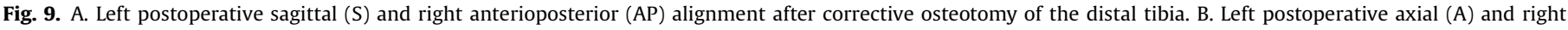
anteroposterior (AP) alignment after corrective osteotomy of the proximal humerus.

Please cite this article in press as: $\mathrm{H}$. Hoekstra, et al., Corrective limb osteotomy using patient specific 3D-printed guides: A technical note, Injury (2016), http://dx.doi.org/10.1016/j.injury.2016.07.021 


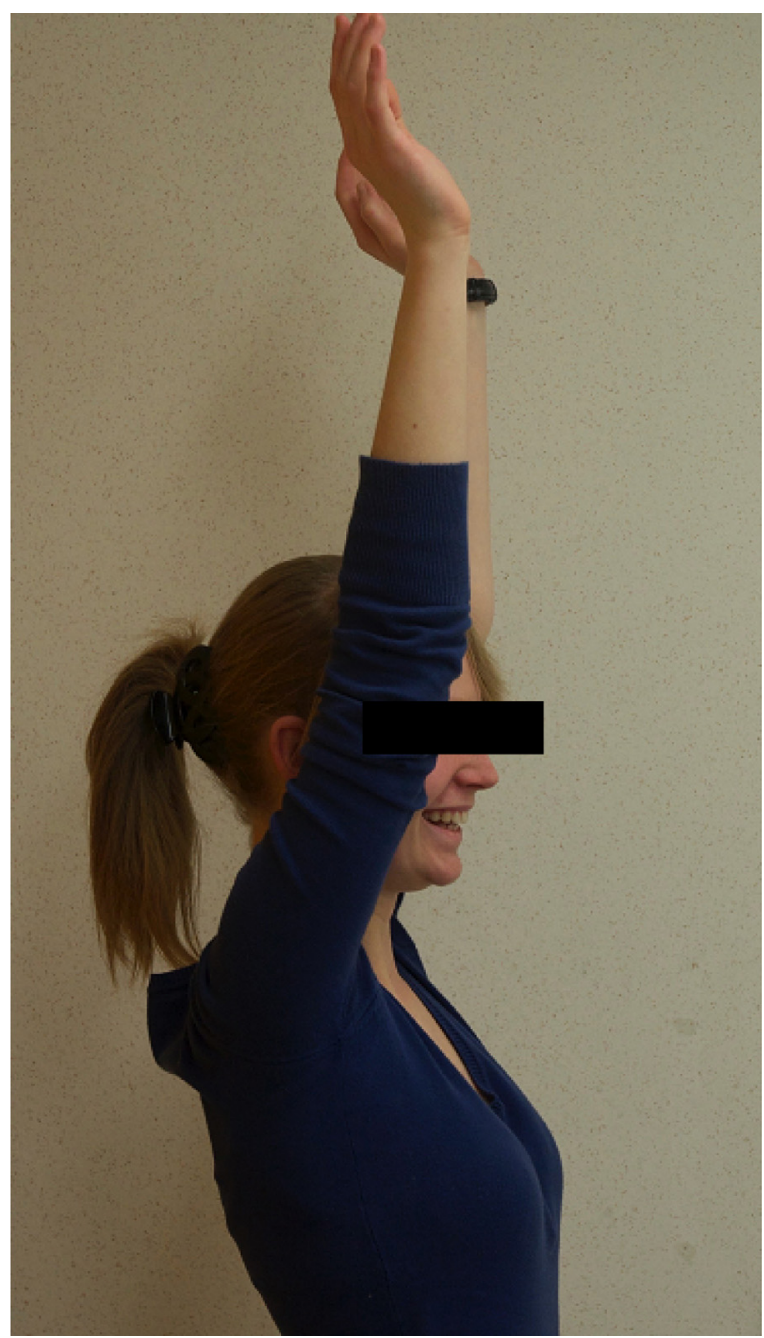

Fig. 10. Good postoperative clinical outcome after corrective osteotomy of the proximal humerus (lefs-sided). (articular osteotomy) with potential long-term complications (e.g. nonunion, functional impairment). Therefore, sometimes it may be appropriate to correct for this during the preoperative process. However, the preoperative correction degree remains guesswork to some extent.

Finally, this technique is relatively expensive in Belgium since preoperative calculations and prints are not fully reimbursed [10].

In conclusion, although not entirely straightforward, this technique is very precise and seems very promising especially in those patients requiring limb corrections in multiple planes. Nevertheless, the surgical skills remain of high importance. Future research should focus on functional outcome in a large patient cohort. Validation of measurements in 3D using standard X-ray images can be an effective strategy to cut the costs.

\section{References}

[1] McGurk M, Amis AA, Potamianos P, Goodger NM. Rapid prototyping techniques for anatomical modeling in medicine. Ann R Coll Surg Engl 1997;79:169-74.

[2] Harris J, Rimell J. Can rapid prototyping ever become a routine feature in general dental practice. Dent Update 2002;29:482-6.

[3] Wagner JD, Baack B, Brown GA, Kelly J. Rapid 3-dimensional prototyping for surgical repair of maxillofacial fractures: a technical note. J Oral Maxillofac Surg 2004;62:898-901.

[4] Brown GA, Firoozbakhsh K, DeCoster TA, Reyna Jr. JR, Moneim M. Rapid prototyping: the future of trauma surgery. J Bone Joint Surg Am 2003;85:4955 .

[5] Kunz M, Ma B, Rudan JF, Ellis RE, Pichora DR. Image-guided distal radius osteotomy using patient-specific instrument guides. J Hand Surg Am 2013:38:1618-24.

[6] Schweizer A, Fürnstahl P, Nagy L. Three-dimensional correction of distal radius intra-articular malunions using patient-specific drill guides. J Hand Surg Am 2013:38:2339-47.

[7] Takeyasu Y, Oka K, Miyake J, Kataoka T, Moritomo H, Murase T. Preoperative computer simulation-based three-dimensional corrective osteotomy for cubitus varus deformity with use of a custom-designed surgical device. J Bone Joint Surg Am 2013:95:1731-9.

[8] Victor J, Premanathan A. Virtual 3D planning and patient specific surgica guides for osteotomies around the knee: a feasibility and proof-of-concept study. Bone Joint J 2013;95:153-8.

[9] Rengier F, Mehndiratte A, von Tengg-Kobligk H, Zechmann CM, Unterhinninghofen R, Kauczor HU, et al. 3D printing based on imaging data: review of medical applications. Int J CARS 2010;5:335-41.

[10] Smeets B, Nijs S, Nderlita M, Vandoren C, Hoekstra H. Health care usage and related costs in fibular plating for AO type 44-B ankle fractures in a Belgian university hospital: an exploratory analysis. J Foor Ankle Surg 2016;2016. 